\title{
RUPTURED SINUS OF VALSALVA ANEURYSM (SOVA): A CASE REPORT
}

\author{
Fakher-e-Fayaz, Lubna Shaheen*, Muhammad Adnan**, Hana Khurshid** \\ Armed Forces Institute of Cardiology/National Institute of Heart Disease (AFIC/NIHD)/National University of Medical Sciences (NUMS) \\ Rawalpindi Pakistan, *Queen Elizabeth Hospital, Birmingham UK, **Islamabad Medical And Dental College, Islamabad Pakistan
}

\begin{abstract}
Sinus of Valsalva Aneurysm is a rare anomaly of the cardiovascular system. It may be acquired or congenital and may also be associated with another cardiac lesion. Acute onset of dyspnea is experienced when the aneurysm ruptures. Diagnosis is reached by echocardiography and Cardiac MRI. The treatment is surgical repair. This paper presents the case of a 24-year old male with acute onset of symptoms caused by a ruptured sinus of Valsalva Aneurysm.
\end{abstract}

Keywords: Non coronary sinus, Ruptured sinus of valsalva aneurysm, Right atrium.

This is an Open Access article distributed under the terms of the Creative Commons Attribution License (http://creativecommons.org/licenses/by/4.0), which permits unrestricted use, distribution, and reproduction in any medium, provided the original work is properly cited.

\section{BACKGROUND}

The Sinus of Valsalva Aneurysm (SOVA) is a rare congenital anomaly of the cardiovascular system with one percent prevalence rate ${ }^{1}$. Moreover, another study shows that Sinus of Valsalva Aneurysm is responsible for $0.78 \%$ of congenital open-heart operations ${ }^{2}$. The right Sinus of Valsalva is affected by aneurysmal dilation in 70 percent of cases, 25 percent involves the non-coronary sinus, while $5 \%$ affects the Left Coronary Sinus ${ }^{3}$.

Inmostcases, it is attributed to a congenital deficiency of musculo-elastictissue in the aortic tunica media and annulus fibrosus of the aortic valve. The fatal complication of SOV Aisarupture which results in intracardiacs hunting, Cardiac Tamponade. If it involves the pericardial space or Acute Myocardial Infarction4. Diagnosisis made on Transthoracic Echocardiography. However, ifitis inconclusive orasurgery is planned then Transesophageal Echocardiography is a must ${ }^{5}$. Aortic Angiography \& CT Angiography can be considered 6 . Another option may be cardiac MRI7. We describethe case of ruptured SOVA with communication between thenon-coronary sinus and therightatrium.

Correspondence: Dr Fakher-e-Fayaz, Department of Cardiac Anaesthesia, AFIC/NIHD Rawalpindi Pakistan

\section{CASE REPORT}

A case presented with7, days history of dyspnea and acute chest pain in a peripheral hospital. Treatment started on the lines of chest infection. Afterwards, he developed progressive worsening of breathlessness associated with abdominal distention, generalized edema and jaundice. On arrival in a tertiary care hospital he was conscious, oriented but feeling very lethargic. Cross-examination showed that he was hemodynamically stable, with sacral and pedal edema, and a raised jugular venous pulse. The cardiovascular system examination showed a bounding pulse, while a continuous machinery murmur was heard at the center of the chest. There was abdominal distention, with positive fluid thrill and a palpable liver, $4 \mathrm{~cm}$ below the costal margins. Chest percussion showed a dull note bilaterally with reduction of air entry up to mid zones.

\section{Investigation}

On Echocardiography he was diagnosed with ruptured SOVA with communication between the non-coronary sinus and the right atrium. The right ventricle was volume 3 overloaded and grossly distended.

The non-coronary sinus was dilated with a windsock-like structure protruding into the right atrium with an opening at the end (figure-1). The color flow doppler confirmed significant flow 
into the right atrium just above the tricuspid valve (figure).

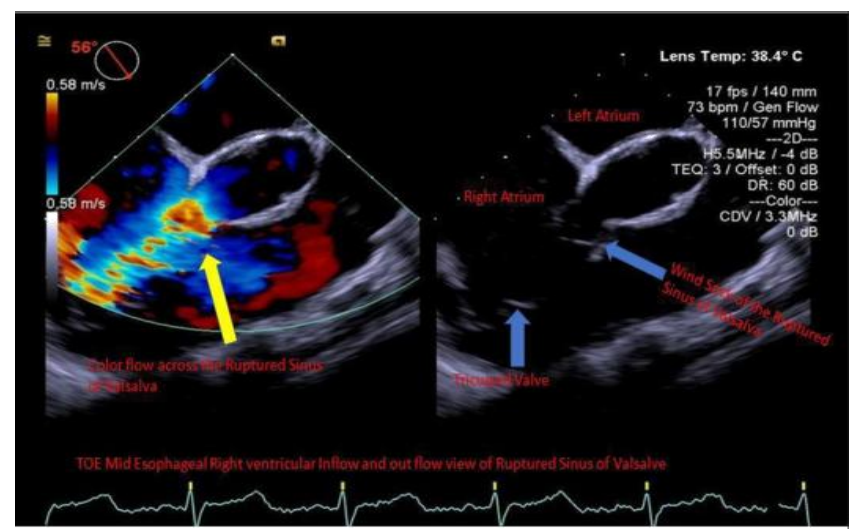

Figure: TOE Image showing dilation of the noncoronary sinus with protrusion of a windsock-like structure from the non-coronary sinus area into the right atrium. A case presented with 7 days history of dyspnea and acute chest pain in a peripheral hospital.

\section{Treatment and Outcome}

By employing “The Double Exposure Technique" the defect was repaired with PTFE patch and he was discharged home on 7 th post-op day.

\section{DISCUSSION}

Ruptured Sinus of Valsalva Aneurysm (SOVA) is more common in males than it is in females. Studies have shown that the average age of rupture in males is 34 years $^{8}$. Most cases of ruptured sinus of Valsalva aneurysm are congenital rather than acquired. Factors that cause acquired SOVA include Syphilis, Infective Endocarditis, Trauma, Atherosclerosis, Marfans Syndrome, Medial Cystic Necrosis and Connective Tissue Disorders. Acute rupture of SOVA is signaled by rapid onset of severe retrosternal chest pain or symptoms of heart failure.

The uniqueness of this case is explained thus: the Aneurysm of the sinus of Valsalva is a rare disease. In most cases of sinus of Valsalva Aneurysm, the right coronary sinus ruptures into the right ventricle, while a few originate from non-coronary sinus and rupture into the right atrium $^{6}$. The left coronary sinus aneurysm is rare $^{9}$. Our case is focused on SOVA originating from the non-coronary sinus. Studies show that ${ }^{4}$. Only $25 \%$ of aneurysms originate from the noncoronary sinus with wide prevalence in Asian males $^{3}$. Presently, surgical repair of a ruptured SOVA has a ten-year survival rate with possibility of aortic regurgitation post-operatively ${ }^{10}$.

\section{CONCLUSION}

Acute onset dyspnea may be as a result of a ruptured SOVA. The best choice of treatment is surgery.

\section{CONFLICT OF INTEREST}

This study has no conflict of interest to be declared by any author.

\section{REFERENCES}

1. Wells T, Byrd B, Neirste D, Fleurelus C. Sinus of Valsalva aneurysm with rupture into the interventricular septum and left the ventricular cavity. Circulation 1999; 100(17): 1843-44.

2. Dong C, WU, Tang Y. Ruptured sinus of Valsalva aneurysm: a Beijing experience. Annals Thoracic Surg 2002; 74(5): 1621-24.

3. Moustafa S, Mookadam F, Cooper L, Adam G, Zehr K, Stulak J. Sinus of Valsalva aneurysms-47 years of a single center experience and systematic overview of published reports. Amer J Cardiol 2007; 99(8): 1159-64.

4. Meijers A, van der Schoot MJ, Maat LP, Bruning TA, Geleijnse ML, Bogers AJ. Cardiac tamponade due to a ruptured aneurysm of the sinus of Valsalva. J Card Surg 2008; 23(8): 256-58.

5. Smedema JP, Freeman V, Brink J. Aneurysm of the left aortic sinus causing acute myocardial infarction. Circulation 2011; 67(5): 1151-54.

6. Yang $Y$, Zhang L, Wang X, Qing LU, Lin HE, Wang J, et al. Echocardiographic diagnosis of rare pathological patterns of the sinus of Valsalva Aneurysm. Plos One 2017; 12(3): e0173122.

7. Hanna MF, Malguria N, Saboo SS, Jordan KG, Landay $M$, Ghoshhajra BB. Suhny Abbara Cross-sectional imaging of sinus of Valsalva aneurysms: lessons learned. Diagn Interv Radiol 2017; 23(5): 339-46.

8. Sakakibara S, Konno S. Congenital aneurysm of the sinus of Valsalva associated with ventricular septal defect: anatomical aspects. 1968 Am Heart J 1968; 75(5): 595-03.

9. Viktorsson TV, Arnorsson T, Sigurdsson MI, Sverrisson JT, Gudbjartsson T. A giant unruptured aneurysm of the sinus of Valsalva together with ectasia of the left coronary artery. Ann Thorac Surg 2011; 92(1): 354-56.

10. Jung SH, Yun TJ, Im YM, et al. Ruptured sinus of Valsalva aneurysm: transaortic repair may cause sinus of Valsalva distortion and aortic regurgitation. J Thorac Cardiovasc Surg 2008; 135(5): 1153-58. 